\title{
Structural Diversity and Properties of M(II) Coordination Compounds \\ Constructed by 3-Hydrazino-4-amino-1,2,4-triazole Dihydrochloride as Starting Material
}

\author{
Caixia Xu ${ }^{\text {a }}$, Jianguo Zhang ${ }^{\mathrm{a}, *}$, Xin Yin ${ }^{\mathrm{a}}$, Zhenxuan Cheng ${ }^{\mathrm{b}}$ \\ ${ }^{a}$ State Key Laboratory of Explosion Science and Technology, Beijing Institute of Technology, Beijing 100081, PR China \\ ${ }^{\mathrm{b}}$ China north industries group corporation, Beijing 100821, PR China
}

\section{Supporting Information}

List and Capital of Tables

Table S1. Crystal data and structure refinements for compounds 1-12

Table S2. Hydrogen bonds for compounds 1-3, 5, 8, 9 and12.

\section{List and Capital of Figures}

Figure S1. Packing diagram of the 3D structure in $\mathbf{1}$ along $a b$-plane.

Figure S2. Packing diagram of the 3D structure in $\mathbf{2}$ along $a b$-plane.

Figure S3. Packing diagram of the 3D structure in 3 along $a b$-plane.

Figure S4. Packing diagram of the 3D structure in 5 along $b c$-plane.

Figure S5. Packing diagram of the 3D structure in 8 along $a b$-plane.

Figure S6. Packing diagram of the 3D structure in 9 along $a b$-plane.

Figure S7. Packing diagram of the 3D structure in $\mathbf{1 2}$ along $a b$-plane.

Figure S8. DSC curves of compounds 1-12 with the heating rate of $5{ }^{\circ} \mathrm{C} \min ^{-1}$. 
Table S1. Crystal data and structure refinements for compounds 1-12

\begin{tabular}{|c|c|c|c|c|c|c|}
\hline & 1 & 2 & 3 & 4 & 5 & 6 \\
\hline CCDC No. & 1408569 & 1408570 & 1408571 & 1408572 & 1408573 & 1408574 \\
\hline Formula & $\mathrm{C}_{4} \mathrm{H}_{12} \mathrm{MnN}_{12} \mathrm{Cl}_{2} \mathrm{O}_{8}$ & $\mathrm{C}_{6} \mathrm{H}_{18} \mathrm{MnCl}_{2} \mathrm{~N}_{18} \mathrm{O}_{4}$ & $\mathrm{C}_{12} \mathrm{H}_{45} \mathrm{Co}_{3} \mathrm{Cl}_{6} \mathrm{~N}_{24} \mathrm{O}_{34.50}$ & $\mathrm{C}_{6} \mathrm{H}_{18} \mathrm{CoCl}_{2} \mathrm{~N}_{18} \mathrm{O}_{4}$ & $\mathrm{C}_{6} \mathrm{H}_{28} \mathrm{Co}_{2} \mathrm{Cl}_{4} \mathrm{~N}_{12} \mathrm{O}_{6}$ & $\mathrm{C}_{12} \mathrm{H}_{45} \mathrm{Ni}_{3} \mathrm{Cl}_{6} \mathrm{~N}_{24} \mathrm{O}_{34.50}$ \\
\hline FW & 482.10 & 532.22 & 1467.21 & 536.21 & 624.06 & 1466.55 \\
\hline Temp (K) & $298(2)$ & $296(2)$ & 293(2) & $298(2)$ & $296(2)$ & $298(2)$ \\
\hline Crystal system & Monoclinic & Trigonal & Monoclinic & Trigonal & Monoclinic & Monoclinic \\
\hline Space group & $P 2(1) / c$ & P3 & $C 2 / m$ & P3 & $P 2(1) / n$ & $C 2 / m$ \\
\hline$Z$ & 4 & 1 & 4 & 1 & 2 & 4 \\
\hline$a(\AA)$ & $10.6001(8)$ & $9.5156(2)$ & $21.4911(11)$ & $9.4129(7)$ & $9.9407(12)$ & $21.4322(18)$ \\
\hline$b(\AA)$ & $19.3309(18)$ & $9.5156(2)$ & $23.2808(11)$ & $9.4129(7)$ & $8.9765(11)$ & $23.054(2)$ \\
\hline$c(\AA)$ & $7.9310(6)$ & $6.1577(3)$ & $12.2729(8)$ & $6.1506(4)$ & $13.6105(17)$ & $12.1309(11)$ \\
\hline$\alpha\left(^{\circ}\right)$ & 90 & 90 & 90 & 90 & 90 & 90 \\
\hline$\beta\left(\left(^{\circ}\right)\right.$ & $104.928(2)$ & 90 & $119.763(2)$ & 90 & $102.749(2)$ & $119.618(2)$ \\
\hline$\gamma\left({ }^{\circ}\right)$ & 90 & 120 & 90 & 120 & 90 & 90 \\
\hline$V\left(\AA^{3}\right)$ & $1570.3(2)$ & $482.86(3)$ & $5330.5(5)$ & $471.95(6)$ & $1184.6(3)$ & $5210.7(8)$ \\
\hline$D_{\mathrm{c}}\left(\mathrm{g} \cdot \mathrm{cm}^{-3}\right)$ & 2.039 & 1.830 & 1.828 & 1.887 & 1.750 & 1.869 \\
\hline$F(000)$ & 972 & 271 & 2976 & 273 & 636 & 2988 \\
\hline Reflections collected & 7765 & 4018 & 9023 & 2966 & 6578 & 13210 \\
\hline Reflections unique & 2767 & 1407 & 4810 & 1489 & 2113 & 4716 \\
\hline$R_{\text {int }}$ & 0.0465 & 0.0163 & 0.0990 & 0.0257 & 0.0237 & 0.0494 \\
\hline Parameters & 244 & 118 & 439 & 100 & 181 & 444 \\
\hline Goodness-of-fit on $F^{2}$ & 1.048 & 1.033 & 1.052 & 1.061 & 1.062 & 1.130 \\
\hline$R_{1}, w R_{2}[I>2 \sigma(I)]$ & $0.0416,0.0942$ & $0.0174,0.0446$ & $0.1202,0.2755$ & $0.0284,0.0629$ & $0.0227,0.0571$ & $0.0845,0.2575$ \\
\hline$R_{1}, w R_{2}$ (all data) & $0.0649,0.1058$ & $0.0176,0.0447$ & $0.2160,0.3304$ & $0.0323,0.0656$ & $0.0260,0.0591$ & $0.1209,0.2862$ \\
\hline \multirow[t]{2}{*}{$\Delta \rho_{\max }, \Delta \rho_{\min }\left(\mathrm{e} \cdot \AA^{-3}\right)$} & $0.493,-0.567$ & $0.152,-0.201$ & $1.164,-0.675$ & $0.375,-0.304$ & $0.301,-0.235$ & $1.997,-1.234$ \\
\hline & 7 & 8 & 9 & 10 & 11 & 12 \\
\hline CCDC No. & 1408577 & 1408576 & 1408575 & 1044968 & 1044965 & 1408568 \\
\hline Formula & $\mathrm{C}_{6} \mathrm{H}_{18} \mathrm{NiCl}_{2} \mathrm{~N}_{18} \mathrm{O}_{4}$ & $\mathrm{C}_{4} \mathrm{H}_{30} \mathrm{Cl}_{4} \mathrm{~N}_{12} \mathrm{Ni}_{2} \mathrm{O}_{16}$ & $\mathrm{C}_{4} \mathrm{H}_{28} \mathrm{Cl}_{4} \mathrm{~N}_{12} \mathrm{Ni}_{2} \mathrm{O}_{24}$ & $\mathrm{C}_{4} \mathrm{H}_{12} \mathrm{ZnN}_{12} \mathrm{Cl}_{2} \mathrm{O}_{8}$ & $\mathrm{C}_{6} \mathrm{H}_{18} \mathrm{ZnCl}_{2} \mathrm{~N}_{18} \mathrm{O}_{4}$ & $\mathrm{C}_{16} \mathrm{H}_{48} \mathrm{Cd}_{5} \mathrm{Cl}_{10} \mathrm{~N}_{48} \mathrm{O}_{16}$ \\
\hline
\end{tabular}




\begin{tabular}{|c|c|c|c|c|c|c|}
\hline Fw & 535.99 & 761.62 & 887.60 & 492.53 & 542.65 & 2085.52 \\
\hline Temp (K) & $298(2)$ & $293(2)$ & $298(2)$ & $298(2)$ & $296(2)$ & $298(2)$ \\
\hline Crystal system & Trigonal & Triclinic & Monoclinic & Monoclinic & Trigonal & Tetragonal \\
\hline Space group & $P 3$ & $P-1$ & $P 2(1) / c$ & $C 2 / c$ & $P 3$ & $I 4(1) / a$ \\
\hline$Z$ & 1 & 1 & 2 & 4 & 1 & 4 \\
\hline$a(\AA)$ & $9.3258(4)$ & $7.6985(5)$ & $9.1370(8)$ & $10.5959(8)$ & $9.4540(9)$ & $20.4062(17)$ \\
\hline$b(\AA)$ & $9.3258(4)$ & $8.6832(6)$ & $15.4381(13)$ & 19.5181(17) & $9.4540(9)$ & $20.4062(17)$ \\
\hline$c(\AA)$ & $6.0815(2)$ & $10.9672(11)$ & $10.6689(9)$ & $7.9310(6)$ & $6.1473(11)$ & $15.3975(12)$ \\
\hline$\alpha\left(^{\circ}\right)$ & 90 & $71.1180(10)$ & 90 & 90 & 90 & 90 \\
\hline$\beta\left(^{\circ}\right)$ & 90 & $72.4340(10)$ & $103.544(2)$ & $104.956(2)$ & 90 & 90 \\
\hline$\gamma\left({ }^{\circ}\right)$ & 120 & $86.012(2)$ & 90 & 90 & 120 & 90 \\
\hline$V\left(\AA^{3}\right)$ & $458.05(3)$ & $661.01(9)$ & $1463.1(2)$ & $1544.7(2)$ & $475.82(11)$ & $6411.7(9)$ \\
\hline$D_{\mathrm{c}}\left(\mathrm{g} \cdot \mathrm{cm}^{-3}\right)$ & 1.943 & 1.913 & 2.015 & 2.118 & 1.894 & 2.160 \\
\hline$F(000)$ & 274 & 390 & 904 & 992 & 276 & 4072 \\
\hline Reflections collected & 2779 & 4021 & 7184 & 3896 & 3912 & 13569 \\
\hline Reflections unique & 1416 & 2327 & 2582 & 1362 & 1228 & 2816 \\
\hline$R_{\text {int }}$ & 0.0358 & 0.0432 & 0.0293 & 0.0796 & 0.0162 & 0.0654 \\
\hline Parameters & 94 & 173 & 208 & 160 & 118 & 252 \\
\hline Goodness-of-fit on $F^{2}$ & 1.008 & 1.037 & 1.046 & 1.091 & 1.122 & 1.025 \\
\hline$R_{1}, w R_{2}[I>2 \sigma(I)]$ & $0.0357,0.0792$ & $0.0413,0.1014$ & $0.0318,0.0774$ & $0.0729,0.1954$ & $0.0151,0.0383$ & $0.0465,0.1067$ \\
\hline$R_{1}, w R_{2}$ (all data) & $0.0439,0.0829$ & $0.0462,0.1058$ & $0.0446,0.0846$ & $0.0996,0.2108$ & $0.0152,0.0383$ & $0.0917,0.1333$ \\
\hline$\Delta \rho_{\max }, \Delta \rho_{\min }\left(\mathrm{e} \cdot \AA^{-3}\right)$ & $0.721,-0.434$ & $0.650,-0.832$ & $0.488,-0.567$ & $0.161,-0.214$ & $1.258,-0.923$ & $1.255,-0.924$ \\
\hline
\end{tabular}


Table S2. Hydrogen bonds for compounds 1-3, 5, 8, 9 and12.

\begin{tabular}{|c|c|c|c|c|}
\hline $\mathrm{D}-\mathrm{H} \cdots \mathrm{A}$ & D-H $(\AA)$ & $\mathrm{H} \cdots \mathrm{A}(\AA)$ & $\mathrm{D} \cdots \mathrm{A}(\AA)$ & $\mathrm{D}-\mathrm{H} \cdots \mathrm{A}\left({ }^{\circ}\right)$ \\
\hline \multicolumn{5}{|l|}{1} \\
\hline N4--H4B $\cdots \mathrm{O} 3^{[\mathrm{i}]}$ & 0.8900 & 2.2800 & $3.015(4)$ & 140.00 \\
\hline 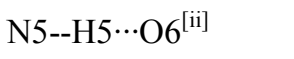 & 0.8600 & 2.5400 & $3.063(5)$ & 120.00 \\
\hline N6--H6A $\cdots \mathrm{O}^{[\mathrm{iii}]}$ & 0.9000 & 2.4200 & $3.308(4)$ & 169.00 \\
\hline 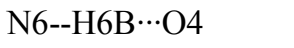 & 0.9000 & 2.3100 & $3.176(5)$ & 161.00 \\
\hline N10--H10A $\cdots \mathrm{O}^{[\mathrm{iii}]}$ & 0.8900 & 2.5200 & $3.123(5)$ & 126.00 \\
\hline N10--H10A $\cdots O 7^{[i v]}$ & 0.8900 & 2.2800 & $3.043(5)$ & 144.00 \\
\hline N10--H10B $\cdots \mathrm{O} 2^{[\mathrm{v}]}$ & 0.8900 & 2.2800 & $3.076(5)$ & 148.00 \\
\hline 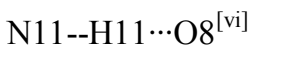 & 0.8600 & 2.3300 & $3.028(5)$ & 139.00 \\
\hline N12--H12B $\cdots \mathrm{O}^{[\mathrm{vii}]}$ & 0.9000 & 2.0800 & $2.876(5)$ & 148.00 \\
\hline $\mathrm{C} 2--\mathrm{H} 2 \cdots \mathrm{O} 4^{[\mathrm{viii}]}$ & 0.9300 & 2.2400 & $3.057(5)$ & 146.00 \\
\hline $\mathrm{C} 4--\mathrm{H} 4 \cdots \mathrm{O} 1^{[\mathrm{v}]}$ & 0.9300 & 2.5400 & $3.364(5)$ & 148.00 \\
\hline \multicolumn{5}{|c|}{ Symmetry codes: [i] x,1/2-y,1/2+z. [ii] 1-x,-y,1-z. [iii] x,y,-1+z. [iv] -x,-y,-z. [v] -1+x,y,-1+z. [vi] -x,-y,1-z. [vii] -1+x,y,z. [viii] x,y,1+z. } \\
\hline \multicolumn{5}{|l|}{2} \\
\hline $\mathrm{N} 6--\mathrm{H} 2 \cdots \mathrm{O} 1^{[\mathrm{i}]}$ & $0.84(2)$ & $2.57(3)$ & $3.3905(19)$ & $163(3)$ \\
\hline N6--H3 $\cdots 5^{5 i i]}$ & $0.92(2)$ & $2.33(3)$ & $3.217(2)$ & $161(2)$ \\
\hline $\mathrm{N} 5--\mathrm{H} 4 \cdots \mathrm{Cl} 2^{[\mathrm{iii}]}$ & $0.82(4)$ & $2.50(3)$ & $3.2273(14)$ & $148(3)$ \\
\hline $\mathrm{N} 4--\mathrm{H} 6 \cdots \mathrm{Cl} 2^{[\mathrm{iv}]}$ & $0.86(3)$ & $2.61(3)$ & $3.3734(18)$ & $148(3)$ \\
\hline $\mathrm{C} 2-\mathrm{H} 1 \cdots \mathrm{O} 1^{[\mathrm{v}]}$ & $0.907(19)$ & $2.50(2)$ & $3.393(2)$ & $168.4(17)$ \\
\hline \multicolumn{5}{|c|}{ Symmetry codes:[i] 1-x+y,1-x,z. [ii] 1-y,x-y,1+z. [iii] 1+x,y,z. [iv] 1+x,y,-1+z. [v] -x+y,-x,-1+z. } \\
\hline \multicolumn{5}{|l|}{3} \\
\hline N8--H8A $\cdots \mathrm{O}^{[\mathrm{i}]}$ & 0.8900 & 2.4300 & $3.10(4)$ & 132.00 \\
\hline N8--H8B $\cdots \mathrm{O} 14$ & 0.8900 & 2.3500 & $3.15(3)$ & 149.00 \\
\hline N12--H12A $\cdots \mathrm{O} 7^{[\mathrm{ii}]}$ & 0.8900 & 2.2700 & $3.01(4)$ & 140.00 \\
\hline N12--H12B $\cdots \mathrm{O} 11$ & 0.8900 & 2.3100 & $3.19(2)$ & 169.00 \\
\hline O16--H16C ‥O21 & 0.8500 & 1.8200 & $2.667(16)$ & 171.00 \\
\hline O16--H16D $\cdots O 19$ & 0.8500 & 1.9800 & $2.83(2)$ & 171.00 \\
\hline O17--H17D $\cdots \mathrm{O} 22$ & 0.8500 & 1.9400 & $2.79(2)$ & 179.00 \\
\hline
\end{tabular}




\begin{tabular}{|c|c|c|c|c|}
\hline O18--H18C‥O5 & 0.8500 & 2.1400 & $2.99(4)$ & 179.00 \\
\hline 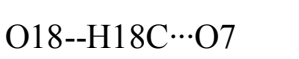 & 0.8500 & 2.5800 & $3.11(4)$ & 122.00 \\
\hline 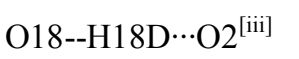 & 0.8500 & 2.3200 & $3.17(6)$ & 178.00 \\
\hline $\mathrm{O} 21--\mathrm{H} 21 \mathrm{C} \cdots \mathrm{O} 8^{[\mathrm{iv}]}$ & 0.8500 & 2.2100 & $2.84(3)$ & 131.00 \\
\hline $\mathrm{O} 22--\mathrm{H} 22 \mathrm{C} \cdots \mathrm{O} 10$ & 0.8500 & 2.2000 & $3.03(3)$ & 166.00 \\
\hline $\mathrm{O} 22--\mathrm{H} 22 \mathrm{C} \cdots \mathrm{O} 10^{[\mathrm{v}]}$ & 0.8500 & 2.4800 & $3.03(3)$ & 123.00 \\
\hline $\mathrm{O} 22--\mathrm{H} 22 \mathrm{D} \cdots \mathrm{O} 14$ & 0.8500 & 1.8900 & $2.72(5)$ & 167.00 \\
\hline $\mathrm{C} 1--\mathrm{H} 1 \cdots \mathrm{O} 10^{[\mathrm{vi}]}$ & 0.9300 & 2.5400 & $3.36(2)$ & 147.00 \\
\hline $\mathrm{C} 2--\mathrm{H} 2 \cdots \mathrm{O} 5^{[\mathrm{viii}]}$ & 0.9300 & 2.1700 & $3.06(3)$ & 158.00 \\
\hline $\mathrm{C} 3--\mathrm{H} 3 \cdots \mathrm{O} 6^{[\mathrm{vi}]}$ & 0.9300 & 2.2100 & $3.10(4)$ & 160.00 \\
\hline $\mathrm{C} 5--\mathrm{H} 5 \cdots \mathrm{O} 8^{[\mathrm{vii}]}$ & 0.9300 & 2.5400 & $3.36(2)$ & 148.00 \\
\hline $\mathrm{C} 6--\mathrm{H} 6 \cdots \mathrm{O} 2^{[\mathrm{iii}]}$ & 0.9300 & 2.4000 & $3.28(5)$ & 157.00 \\
\hline \multicolumn{5}{|c|}{ Symmetry codes: [i] -1/2+x,1/2-y,z. [ii] 1-x,y,1-z. [iii] 1-x,1-y,1-z. [iv] -1/2+x,-1/2+y,-1+z. [v] x,1-y,z. [vi] 1/2-x,1/2-y,1-z. [vii] 1-x,1-y,2-z, [viii] 1/2-x,1/2-y,-z. } \\
\hline \multicolumn{5}{|c|}{ 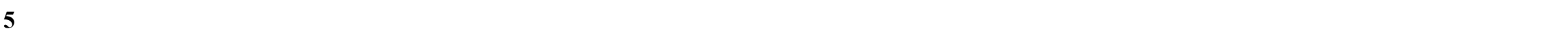 } \\
\hline $\mathrm{N} 4--\mathrm{H} 5 \cdots \mathrm{Cl}^{[\mathrm{i}]}$ & $0.92(3)$ & $2.51(3)$ & $3.335(2)$ & $149(2)$ \\
\hline $\mathrm{N} 4--\mathrm{H} 6 \cdots \mathrm{Cl} 2^{[\mathrm{ii}]}$ & $0.84(3)$ & $2.62(3)$ & $3.345(2)$ & $146(2)$ \\
\hline $\mathrm{N} 5--\mathrm{H} 7 \cdots \mathrm{Cl} 2^{[\mathrm{iii}]}$ & $0.85(3)$ & $2.56(3)$ & $3.3065(19)$ & $147(2)$ \\
\hline $\mathrm{N} 6--\mathrm{H} 8 \cdots \mathrm{Cl} 3^{[\mathrm{iv}]}$ & $0.88(3)$ & $2.47(3)$ & $3.348(2)$ & $178(2)$ \\
\hline $\mathrm{O} 2--\mathrm{H} 10 \cdots \mathrm{Cl} 3^{[\mathrm{v}]}$ & $0.75(3)$ & $2.35(3)$ & $3.088(2)$ & $170(3)$ \\
\hline $\mathrm{O} 2--\mathrm{H} 11 \cdots \mathrm{O} 3^{[\mathrm{i}]}$ & $0.97(3)$ & $1.85(3)$ & $2.810(3)$ & $175(3)$ \\
\hline $\mathrm{O} 3--\mathrm{H} 12 \cdots \mathrm{Cl} 2^{[\mathrm{vi}]}$ & $0.83(4)$ & $2.36(4)$ & $3.183(2)$ & $171(4)$ \\
\hline $\mathrm{O} 3--\mathrm{H} 13 \cdots \mathrm{Cl} 3^{[\mathrm{vii}]}$ & $1.01(4)$ & $2.14(4)$ & $3.149(2)$ & $173(3)$ \\
\hline 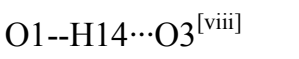 & $0.85(4)$ & $1.97(4)$ & $2.797(3)$ & $165(4)$ \\
\hline \multirow{2}{*}{\multicolumn{5}{|c|}{$\begin{array}{l}\text { Symmetry codes: [i] 1/2+x,1/2-y,-1/2+z. [ii] x,-1+y,z. [iii] 3/2-x,-1/2+y,1/2-z. [iv] 1/2-x,-1/2+y,1/2-z.. [v] 1+x,-1+y,z. [vi] 2-x,1-y,1-z. [vii] 1-x,1-y,1-z. [viii]] x,y,-1+z } \\
\mathbf{8}\end{array}$}} \\
\hline & & & & \\
\hline $\mathrm{N} 5--\mathrm{H} 5 \mathrm{~A} \cdots \mathrm{O} 1^{[\mathrm{i}]}$ & 0.9000 & 2.2900 & $2.892(4)$ & 124.00 \\
\hline N6--H6A $\cdots O 8^{[\mathrm{ii}]}$ & 0.9000 & 2.1900 & $3.048(5)$ & 160.00 \\
\hline N6--H6B $\cdots \mathrm{Cl1}^{[\mathrm{iii}]}$ & 0.9000 & 2.7100 & $3.463(3)$ & 141.00 \\
\hline N6--H6B $\cdots O 6^{[i i i]}$ & 0.9000 & 2.2300 & $2.955(4)$ & 137.00 \\
\hline $\mathrm{O} 5-\mathrm{H} 5 \mathrm{E} \cdots \mathrm{O} 7^{[\mathrm{iv}]}$ & 0.8500 & 1.9200 & $2.775(4)$ & 180.00 \\
\hline
\end{tabular}




\begin{tabular}{|c|c|c|c|c|}
\hline 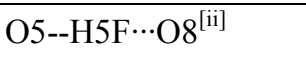 & 0.8500 & 1.9400 & $2.785(4)$ & 179.00 \\
\hline $\mathrm{N} 4--\mathrm{H} 4 \mathrm{~A} \cdots \mathrm{O} 3^{[\mathrm{v}]}$ & 0.8900 & 2.4800 & $3.166(5)$ & 134.00 \\
\hline $\mathrm{N} 4--\mathrm{H} 4 \mathrm{~B} \cdots \mathrm{O} 1^{[\mathrm{iv}]}$ & 0.8900 & 2.3700 & $3.122(5)$ & 142.00 \\
\hline 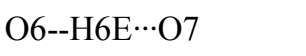 & 0.8500 & 1.8300 & $2.679(4)$ & 174.00 \\
\hline O6--H6F $\cdots \mathrm{Cl1}^{[\mathrm{iii}]}$ & 0.8500 & 2.2900 & $3.138(3)$ & 175.00 \\
\hline $\mathrm{O} 7--\mathrm{H} 7 \mathrm{C} \cdots \mathrm{O} 4^{[\mathrm{vi}]}$ & 0.8500 & 2.0700 & $2.877(4)$ & 158.00 \\
\hline O7--H7D $\cdots \mathrm{Cl1}^{[\mathrm{vi}]}$ & 0.8500 & 2.4200 & $3.262(3)$ & 172.00 \\
\hline 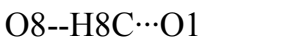 & 0.8500 & 2.0500 & $2.889(4)$ & 169.00 \\
\hline O8--H8D $\cdots \mathrm{Cl1}{ }^{\text {[vii] }}$ & 0.8500 & 2.4600 & $3.300(3)$ & 169.00 \\
\hline \multicolumn{5}{|c|}{ Symmetry codes:[i] x,-1+y,1+z. [ii] -x,1-y,1-z. [iii] 1-x,-y,1-z. [iv] 1-x,1-y,1-z. [v] x,y,1+z. [vi] 1+x,y,z. [vii] x,1+y,z. } \\
\hline \multicolumn{5}{|l|}{9} \\
\hline $\mathrm{N} 4--\mathrm{H} 4 \mathrm{~B} \cdots \mathrm{O} 4^{[\mathrm{i}]}$ & 0.8900 & 2.3100 & $3.103(4)$ & 148.00 \\
\hline 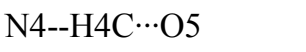 & 0.8900 & 2.5600 & $3.250(4)$ & 135.00 \\
\hline N6--H6A $\cdots \mathrm{O} 3^{[\mathrm{ii}]}$ & 0.9000 & 2.4400 & $3.264(4)$ & 152.00 \\
\hline N6--H6B $\cdots \mathrm{O} 7^{[\mathrm{iii}]}$ & 0.9000 & 2.2900 & $3.187(4)$ & 174.00 \\
\hline O9--H9C ‥O $1^{[\mathrm{iv}]}$ & 0.8500 & 2.1900 & $3.041(4)$ & 176.00 \\
\hline O9--H9D $\cdots \mathrm{O} 12^{[\mathrm{v}]}$ & 0.8500 & 1.9300 & $2.776(4)$ & 175.00 \\
\hline $\mathrm{O} 10--\mathrm{H} 10 \mathrm{C} \cdots \mathrm{O} 3^{[\mathrm{vi}]}$ & 0.8500 & 2.5000 & $3.273(3)$ & 152.00 \\
\hline $\mathrm{O} 10--\mathrm{H} 10 \mathrm{C} \cdots \mathrm{O} 4^{[\mathrm{vi}]}$ & 0.8500 & 2.4200 & $3.188(4)$ & 151.00 \\
\hline O10--H10D $\cdots \mathrm{O} 12^{[\mathrm{vii}]}$ & 0.8500 & 1.9900 & $2.750(3)$ & 149.00 \\
\hline $\mathrm{O} 11--\mathrm{H} 11 \mathrm{C} \cdots \mathrm{O} 2^{[\mathrm{iv}]}$ & 0.8500 & 2.0100 & $2.859(3)$ & 180.00 \\
\hline O11--H11D $\cdots O 3^{[\mathrm{vi}]}$ & 0.8500 & 2.0700 & $2.919(3)$ & 180.00 \\
\hline O12--H12C‥O5 & 0.8500 & 2.1100 & $2.959(4)$ & 173.00 \\
\hline $\mathrm{O} 12--\mathrm{H} 12 \mathrm{D} \cdots \mathrm{O} 1^{[\mathrm{iv}]}$ & 0.8500 & 2.3200 & $3.168(4)$ & 173.00 \\
\hline $\mathrm{C} 2-\mathrm{H} 2 \cdots \mathrm{O} 1^{[\mathrm{vi}]}$ & 0.9300 & 2.5600 & $3.175(4)$ & 124.00 \\
\hline \multicolumn{5}{|c|}{ Symmetry codes: [i] 1-x,-y,-z. [ii] 1-x,-1/2+y,1/2-z. [iii] 1-x,-y,1-z. [iv] 1+x,1/2-y,1/2+z. [v] x,1/2-y,1/2+z. [vi] 1+x,y,z. [vii] 2-x,-1/2+y,1/2-z } \\
\hline \multicolumn{5}{|l|}{12} \\
\hline $\mathrm{N} 4--\mathrm{H} 4 \mathrm{~B} \cdots \mathrm{O} 2^{[\mathrm{i}]}$ & 0.8900 & 2.5200 & $3.23(2)$ & 138.00 \\
\hline $\mathrm{N} 5--\mathrm{H} 5 \cdots \mathrm{Cl1}{ }^{[\mathrm{ii}]}$ & 0.8600 & 2.4000 & $3.167(9)$ & 149.00 \\
\hline N6--H6A $\cdots \mathrm{Cl1}$ & 0.9000 & 2.4200 & $3.303(7)$ & 167.00 \\
\hline
\end{tabular}




\begin{tabular}{|c|c|c|c|c|}
\hline N6--H6B $\cdots \mathrm{O} 2^{[\mathrm{iii}]}$ & 0.9000 & 2.2300 & $2.94(2)$ & 136.00 \\
\hline N10--H10B $\cdots \mathrm{Cl}^{[\mathrm{iv}]}$ & 0.8900 & 2.6500 & $3.340(10)$ & 136.00 \\
\hline $\mathrm{N} 11--\mathrm{H} 11 \cdots \mathrm{Cl} 3^{[\mathrm{v}]}$ & 0.8600 & 2.2300 & $3.004(8)$ & 149.00 \\
\hline $\mathrm{N} 12--\mathrm{H} 12 \mathrm{~A} \cdots \mathrm{O} 1^{[\mathrm{vi}]}$ & 0.9000 & 2.5700 & $3.36(7)$ & 147.00 \\
\hline N12--H12B $\cdots \mathrm{Cl1}{ }^{[\mathrm{vii}]}$ & 0.9000 & 2.6100 & $3.322(7)$ & 136.00 \\
\hline
\end{tabular}




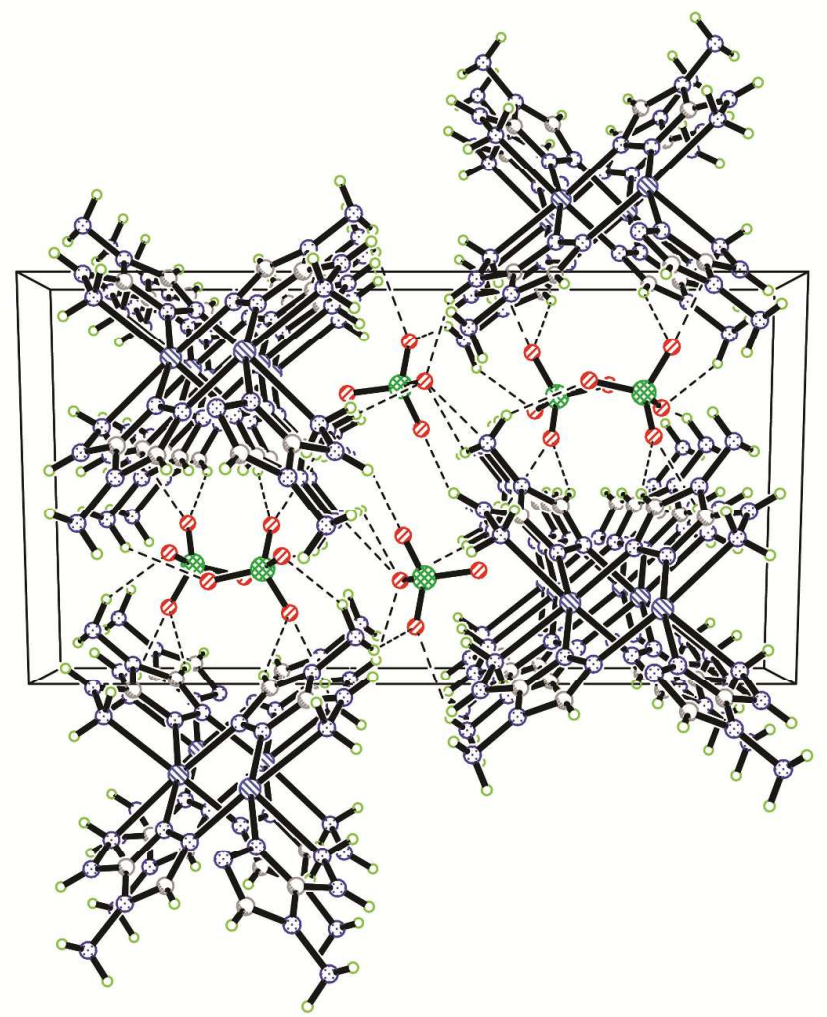

Figure S1. Packing diagram of the 3D structure in $\mathbf{1}$ along $a b$-plane.

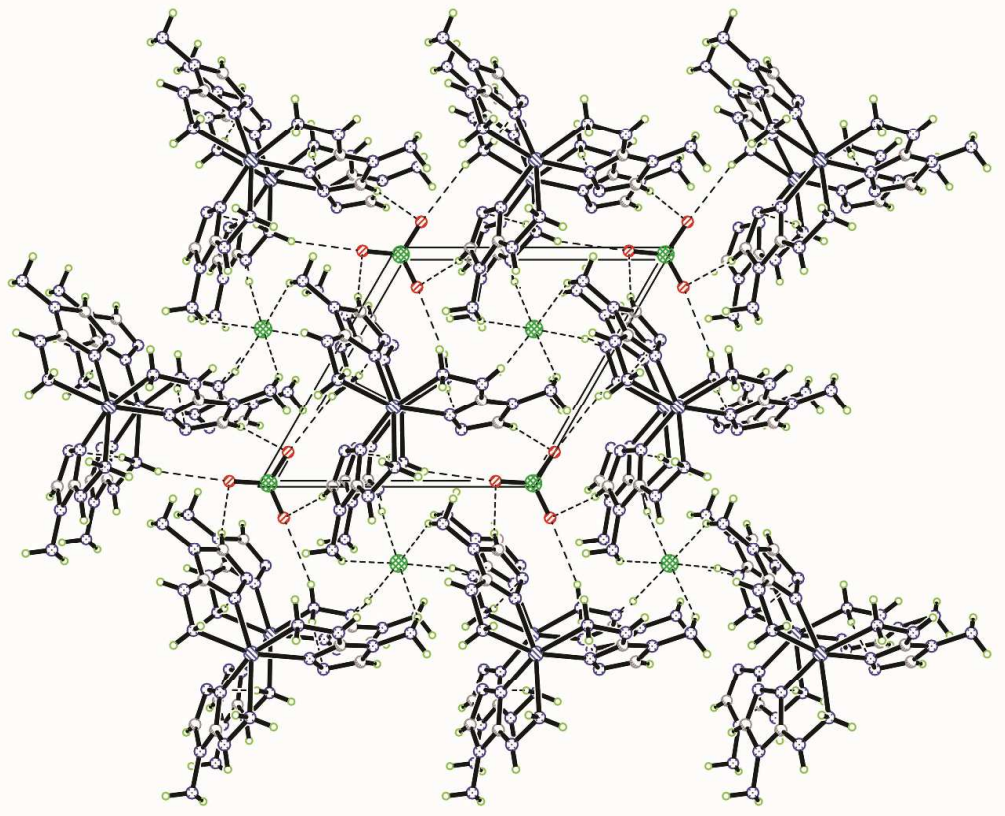

Figure S2. Packing diagram of the 3D structure in $\mathbf{2}$ along $a b$-plane. 


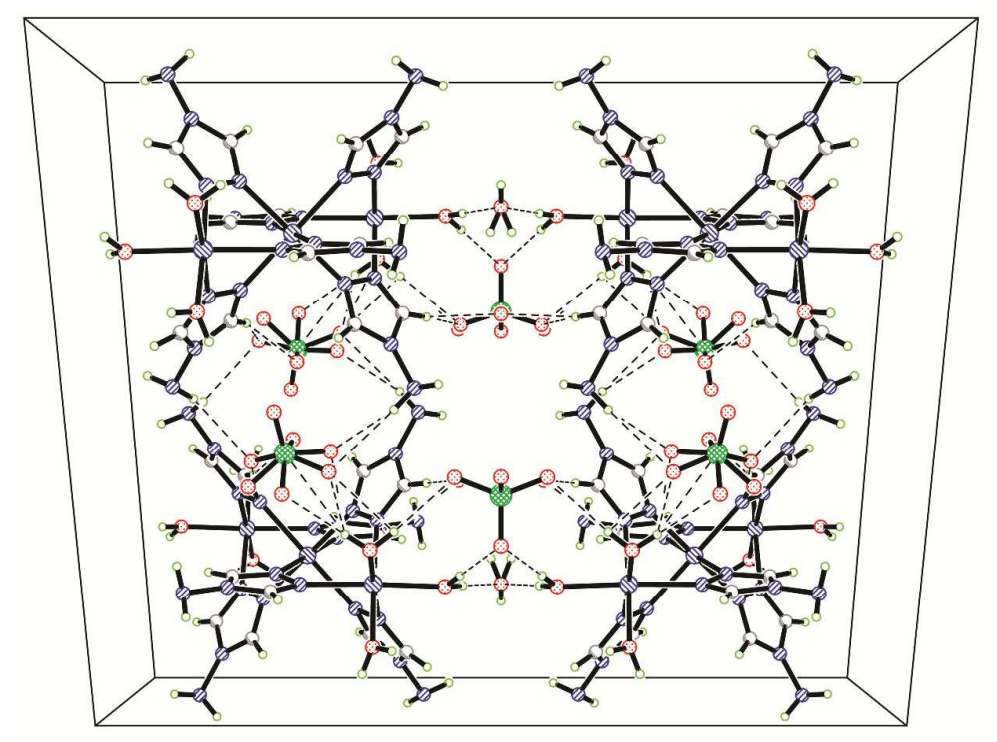

Figure S3. Packing diagram of the 3D structure in $\mathbf{3}$ along $a b$-plane.

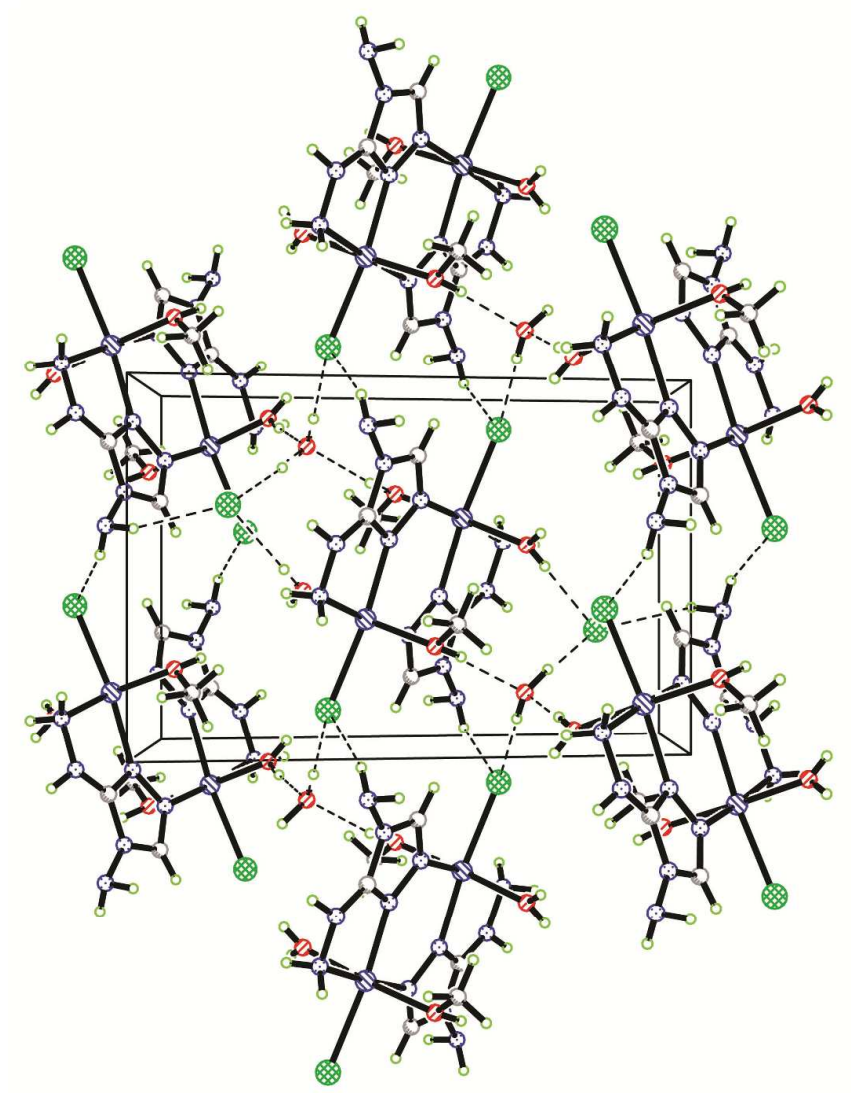

Figure S4. Packing diagram of the 3D structure in $\mathbf{5}$ along $b c$-plane. 


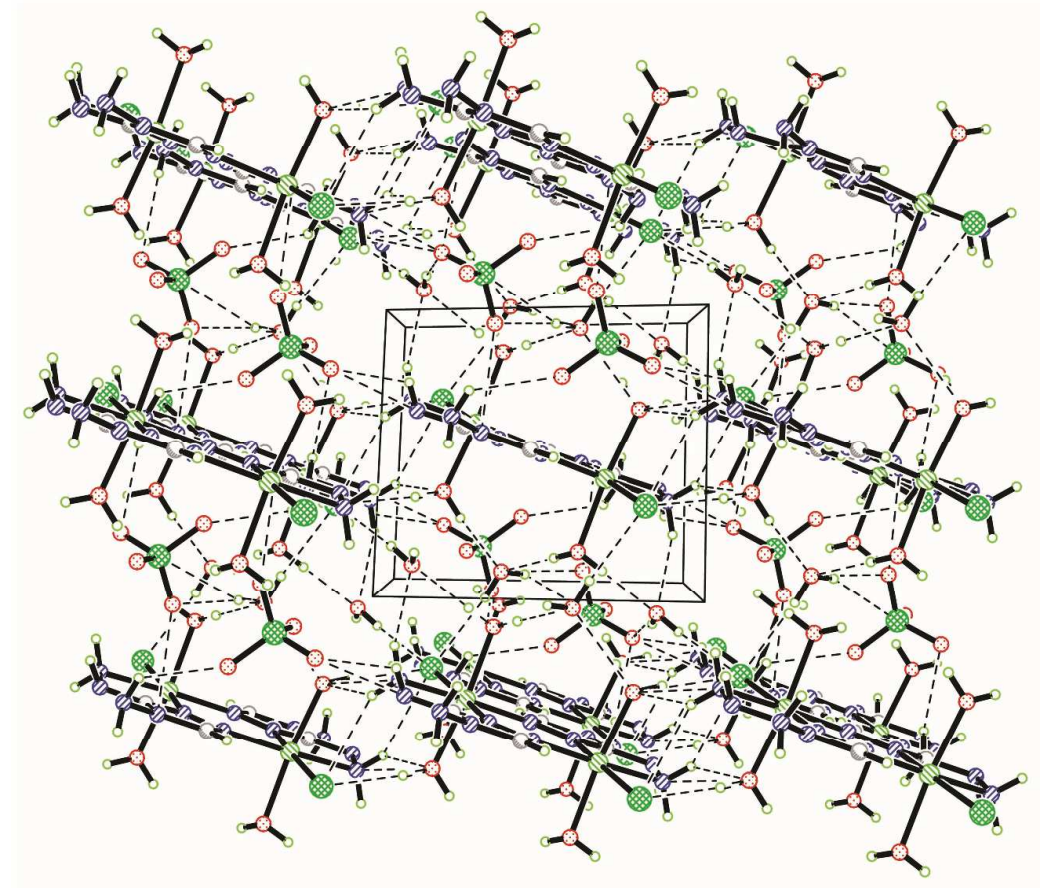

Figure S5. Packing diagram of the 3D structure in $\mathbf{8}$ along $a b$-plane.

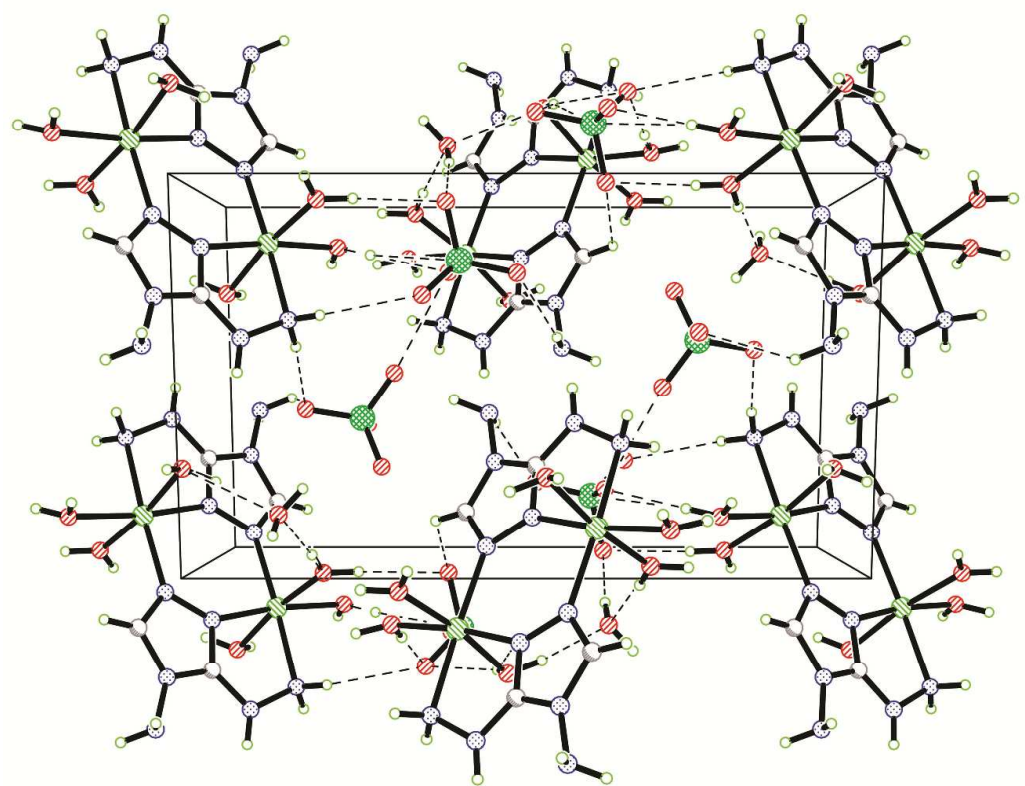

Figure S6. Packing diagram of the 3D structure in 9 along $a b$-plane. 


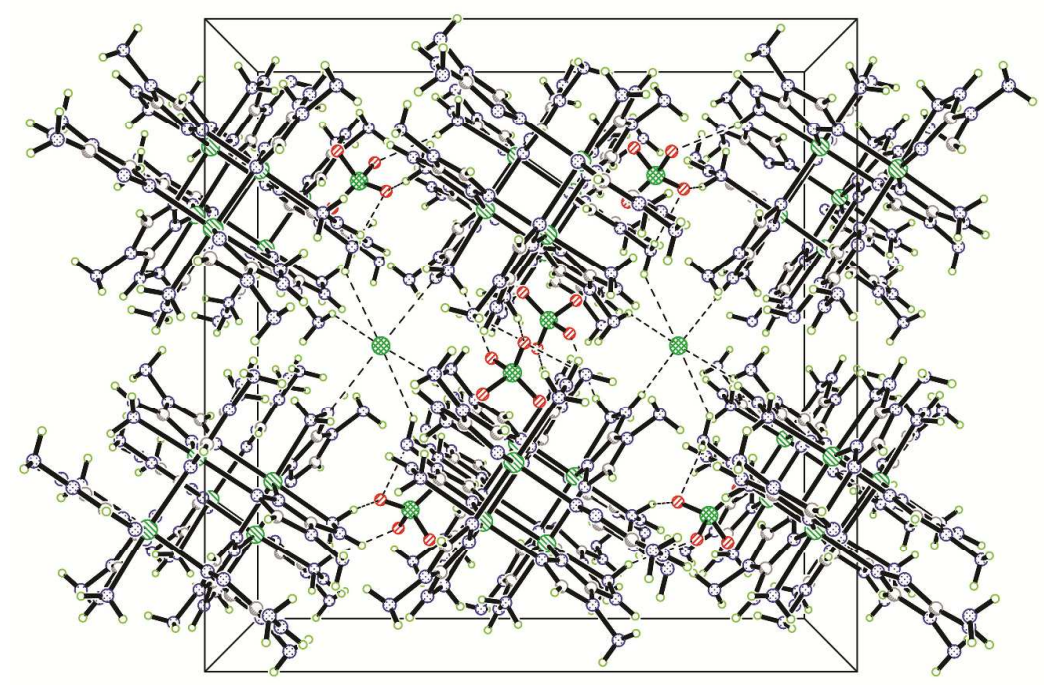

Figure S7. Packing diagram of the 3D structure in $\mathbf{1 2}$ along $a b$-plane.

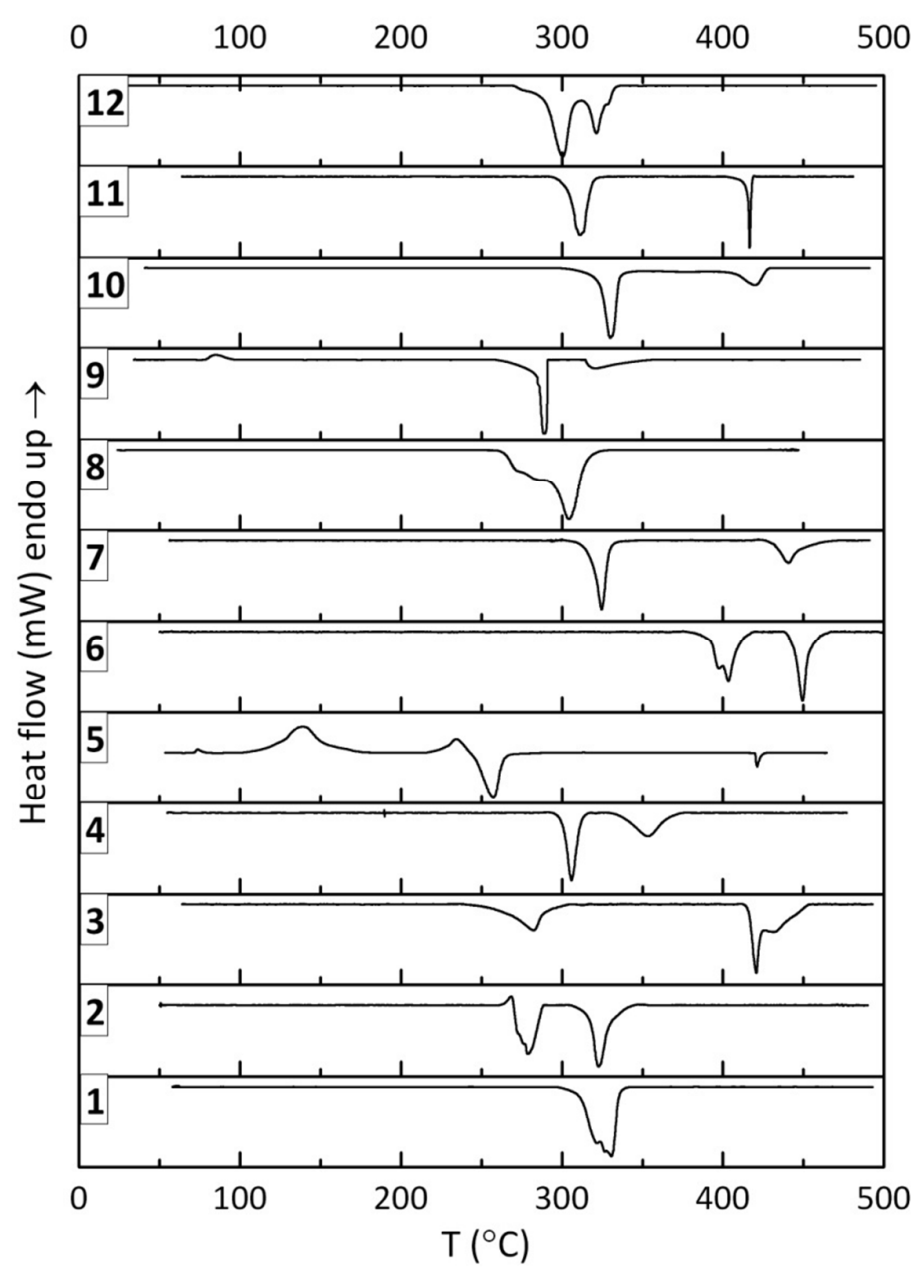

Figure S8. DSC curves of compounds 1-12 with the heating rate of $5{ }^{\circ} \mathrm{C} \mathrm{min}^{-1}$. 